\title{
Amelanismo en Cinclodes moculirostris (Furnariidae), ave endémica del extremo austral de América
}

\section{Amelanism in Cinclodes maculirostris (Furnariidae), endemic bird from austral America}

JUAN C. TORRES-MURA ${ }^{1} \bowtie \&$ OMAR BARROSO

1 torresmura@gmail.com

\section{OPEN ACCESS}

\section{Recibido:}

04/04/2021

Revisado:

27/08/2021

\section{Aceptado:}

$11 / 09 / 2021$

Publicado en línea:

$31 / 12 / 2021$

\section{Editor asociado:}

Dra. Francisca Massardo Sección especial: Reserva de la Biosfera Cabo de Hornos y Parque Marino Islas Diego Ramírez-Paso Drake.

ISSN 0718-686X
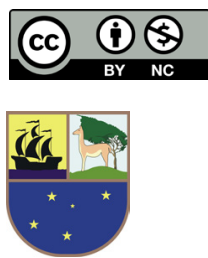

Las aberraciones de coloración de las aves son variaciones en el color del plumaje, piel o el ojo que se presentan de manera esporádica en ciertos individuos. En las aves se han identificado diferentes aberraciones en el color, sin embargo, existe una confusión general cuando se intenta determinar el tipo de aberración (e.g. confusión entre albinismo y leucismo). Esta confusión se debe a una terminología desarrollada en mamíferos, los que difieren en la variedad de pigmentos. También, los tipos de aberración fueron definidos sin conocer con exactitud los mecanismos causantes. Como resultado, las anormalidades observadas en terreno han sido por lo general difíciles de determinar (Davis, 2007). Varios factores han sido propuestos como causa de las aberraciones del plumaje, incluyendo mutaciones genéticas, dieta, edad, enfermedades y parásitos (van Grouw, 2006, 2013). Aunque se acepta que las coloraciones aberrantes reducen la adecuación de los individuos, existen casos, como el de una población de traro (Caracara plancus) en la costa de Argentina, en donde se observa comúnmente una alta frecuencia de individuos leucísticos, sugiriendo una aparente ventaja selectiva relacionada con el hábitat y favorecida por selección natural directamente o por pleiotropía y no por deriva génica (Edelaar et al. 2011).

En este aspecto, existe bastante investigación sobre colores anormales en especies de aves en Europa, Norteamérica y en los últimos años en el Neotrópico 
CÓMO CITAR:

Torres-Mura J.C. \&

Barroso O. (2021).

Amelanismo en Cinclodes

Maculirostris (Furnariidae),

ave endémica del extremo austral de América.

Anales Instituto de la

Patagonia, 49. https:// doi.org/10.22352/

AIP202149020

Contribución de los autores:

JT: Trabajo de terreno y contribuyó de igual manera a la redacción del trabajo.

OB: Trabajo de terreno y contribuyó de igual manera a la redacción del trabajo.

Declaración de interéses:

Los autores declaran no tener conflicto de intereses.

Financiamiento: Sin financiamiento. (e.g. Aráoz et al. 2012; Nemésio, 2001; Piacentini, 2001; Sainz-Borgo et al. 2016), sin embargo, el conocimiento en Chile es aún incipiente (Fuentes \& González-Acuña, 2011). Afortunadamente, con el advenimiento de la fotografía digital diversos registros están disponibles en internet (e.g. www.avesdechile.cl), aunque no siempre las fotografías permiten analizar adecuadamente el tipo de aberración observada (Davis, 2007; van Grouw, 2013).

En esta nota se reporta la observación de un ejemplar de churrete austral (Cinclodes maculirostris Dabbene, 1917) (Aves: Passeriformes) con una coloración anormal.

Como parte del programa de investigación ecológica a largo plazo (LTER por su sigla en inglés) que se desarrolla en la Reserva de Biosfera Cabo de Hornos, entre el 20 y 22 de julio de 2017 se realizó una expedición a la isla Gonzalo del archipiélago Diego Ramírez (Región de Magallanes y Antártica Chilena) que incluyó el estudio de las aves que habitan el ambiente de pastizal de coirón (Poa flabellata), que domina ampliamente la isla llegando a coberturas del 100\% y alcanzando los $2 \mathrm{~m}$ de altura (Schlatter \& Riveros, 1987).

El churrete austral usualmente considerado conespecífico con $C$. antarcticus de las islas Falklands/Malvinas ha sido recientemente reconocido como especie plena por BirdLife International por diferencias morfológicas, como es una coloración más oscura, carencia de rufo en las alas, cola más larga y mancha amarilla en la base del pico, que es más corto (del Hoyo \& Collar, 2016). Además, se caracteriza por la coloración negruzca del dorso, el ahumado oscuro del vientre, la carencia de ceja y banda alar claras y el pico corto y grueso. Esta especie se distribuye en el extremo sur de Sudamérica, en el suroeste de Tierra del Fuego, la isla Navarino y los archipiélagos de Hornos y Diego Ramírez en Chile e isla de los Estados en Argentina. Su hábitat son las playas rocosas especialmente en las cercanías de colonias de aves o mamíferos marinos. Su estado de conservación a nivel global es Casi Amenazada (BirdLife International, 2018), aunque en Chile su estado de conservación no ha sido establecido.

En las cercanías de la radio-estación de la Armada de Chile, donde la vegetación de coirón es más abierta, observamos varios ejemplares de churretes australes que se movían en grupo, y uno de ellos presentó plumas blancas en el lomo, flancos y rectrices. Sus patas, pico y ojos eran de coloración normal (Fig. 1). Posteriormente, el Suboficial de la Armada don Víctor Figueroa nos proporcionó fotografías tomadas en julio de 2018 que muestran el mismo individuo en el mismo lugar. La disposición de plumas blancas en parches con simetría bilateral registrada y la ubicación en regiones corporales como alas, cola y vientre más ojos y pico normales son denominadas "leucismo parcial" (Mahabal et al. 2016; Rodríguez-Ruiz et al. 2017). La ausencia anormal de melanina (eumelanina y feomelanina) en parte del plumaje corresponde a un amelanismo, más específicamente a leucismo parcial; en la literatura zoológica esta condición ha sido llamada albinismo, albinismo parcial, semialbinismo, albinismo incompleto, esquizocroismo, piebaldismo, y fenómeno "Pealea" (Davis, 2007). Corresponde a una mutación genética heredable, recesiva, con error en la deposición de melanina (Mahabal et al. 2016; van Grouw, 2006, 2013). Aunque varía mucho de una 
Fig. 1.- Ejemplar adulto de Cinclodes maculirostris con leucismo parcial.

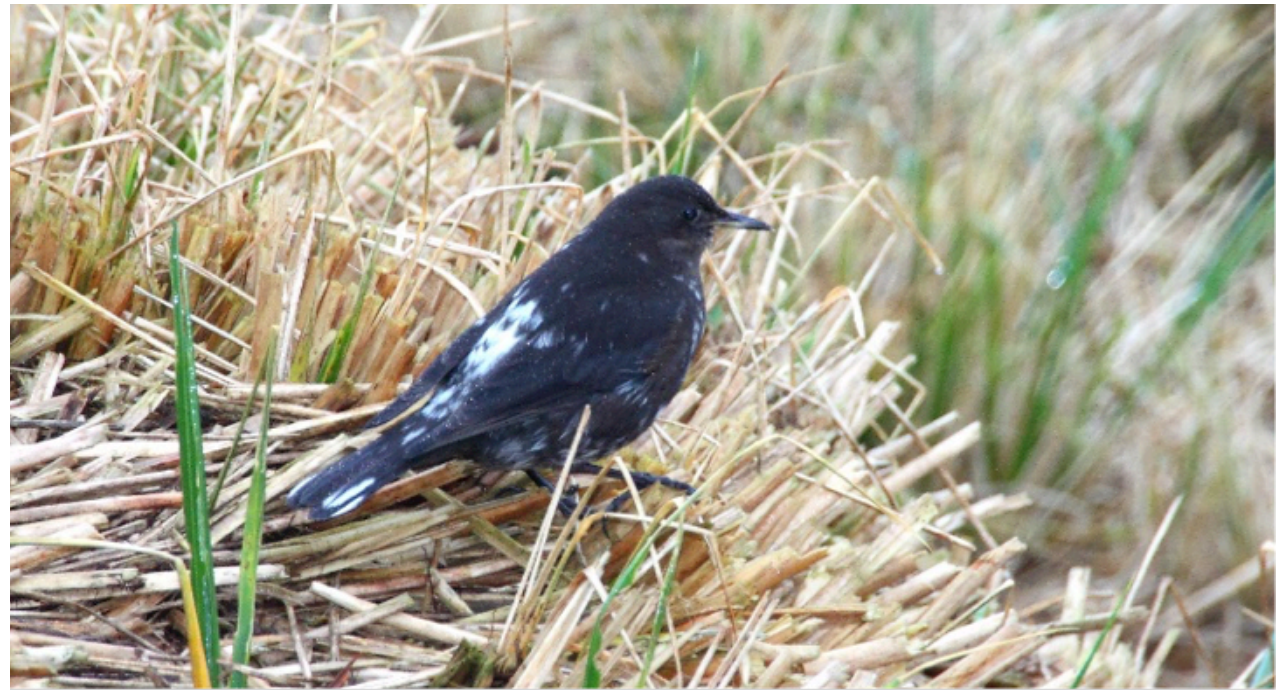

especie a otra, el leucismo es más frecuente en poblaciones urbanas que en rurales y también es más común en poblaciones pequeñas con alto endocruzamiento (Bensch et al. 2000), como podría ser el caso de esta especie de Cinclodes. El leucismo puede ser confundido con el encanecimiento progresivo ya que son difíciles de distinguir en terreno, sin embargo, el blanco causado por el leucismo es irregular pero bilateralmente simétrico, en el encanecimiento progresivo se observan plumas blancas distribuidas aleatoriamente, siendo más frecuentes en la cabeza y los flancos (van Grouw, 2013).

Este hallazgo representa el primer registro de leucismo en el género Cinclodes y el tercero en la familia Furnariidae (Hilty et al. 2013; Lebbin et al. 2006). La documentación de plumajes aberrantes provee de información que ayuda a explicar las causas biológicas de estos plumajes y permite discernir la variación en la frecuencia de aberraciones entre las familias de aves debido a que poblaciones con altas frecuencias de leucismo pueden ser indicadores de endocruzamiento o estrés ambiental (Bensch et al. 2000; Jakubas \& Wojczulanis-Jakubas, 2012) y la situación citada para el traro en Argentina sería una excepción.

Dada la escasez de registros y poco conocimiento en Chile sobre las aves con colores anormales es necesario describir y dar a conocer estos plumajes. Documentar su distribución geográfica y la frecuencia de ocurrencia servirá para determinar, por ejemplo, si estos cambios se deben a procesos naturales o por el contrario aparecen por exposición a contaminantes ambientales (Davis, 2007) u otros factores ambientales de origen antrópico.

\section{AGRADECIMIENTOS}

A la Armada de Chile por el transporte y apoyo logístico. Al personal de la Alcaldía de mar, Isla Gonzalo, en especial a los Suboficiales José Mella y Víctor Figueroa por compartir generosamente su experiencia. La expedición formó parte del Programa de Conservación Biocultural Subantártica del Parque Etnobotánico Omora, la Universidad de Magallanes y el Instituto de Ecología y Biodiversidad de Chile. 


\section{LITERATURA CITADA}

Aráoz, R., Aveldaño, S., \& Ortiz, D. (2012). Casos de plumajes aberrantes en tres especies de aves en Tucumán, Argentina. Acta Zoológica Lilloana, 56, 159-166.

Bensch, S., Hansson, B., Hasselquist, D., y Nielsen, B. (2000). Partial albinism in a semiisolated population of Great Reed Warblers. Hereditas, 133, 167-170.

BirdLife International (2018). IUCN Red List for birds. Downloaded from www.birdlife.org on 19/sept/2018

Davis, J. N. (2007). Color abnormalities in birds: A proposed nomenclature for birders. Birding, 39, 36-46.

del Hoyo, J., \& Collar, N. J. (2016). HBW and BirdLife International Illustrated Checklist of the Birds of the World. Volume

2: Passerines. Lynx Edicions and BirdLife International.

Edelaar, P., Donazar, J., Soriano, M., Santillán, M. A., González-Zevallos, D., García, P., Lisnizer, N., Gatto, A. J., Agüero, M. L., Passera, C. A., Ebert, L. A., Bertellotti, M., Blanco, G., Abril, M., Escudero, G., \& Quintana, F. (2011). Apparent selective advantage of leucism in a coastal population of Southern caracaras (Falconidae). Evolutionary Ecology Research, 13, 187-196.

Fuentes, D., \& González-Acuña, D. (2011). Aberraciones cromáticas del plumaje en aves: nuevos reportes en Chile. Boletín Chileno de Ornitología, 17, 113-121.

Hilty, S. L., Ascanio, D., \& Whittaker, A. (2013). A New Species of Softtail (Furnariidae: Thripophaga) from the delta of the Orinoco river in Venezuela. The Condor, 115, 143-154.

Jakubas, D., y Wojczulanis-Jakubas, K. (2012). Not always black and white: Colour aberrations in the Dovekie. Arctic, 65, 229-232.

Lebbin, D.J., Tori, W.P., \& Bravo, A. (2006). A Ruddy Spinetail Synallaxis rutilans with aberrant plumage. Cotinga, 27, 68-69.

Mahabal, A., van Grouw, H., Sharma, R.M., y Thakur, S. (2016). How common is albinism really? Colour aberrations in Indian birds reviewed. Dutch Birding, 38, 301-309.

Nemésio, A. (2001). Plumagens aberrantes em Emberizidae Neotropicais. Tangara, 1, 39-47.

Piacentini, V. Q. (2001). Novos registros de plumagens aberrantes em Muscicapidae e Emberizidae Neotropicais. Tangara, 1, 183-188

Rodríguez-Ruiz, E. R., Poot-Poot, W. A., Ruiz-Salazar, R., \& Treviño-Carreón, J. (2017). Nuevos registros de aves con anormalidad pigmentaria en México y propuesta de clave dicotómica para la identificación de casos. Huitzil, Revista Mexicana de Ornitología, 18, 57-70.

Sainz-Borgo, C., Ascanio, D., Calcaño, L., López, E., Miranda, J., Rodríguez-Ferraro, A., Ravard, R., Santodomingo, J., Trejo, M., \& van Grouw, H. (2016). Nuevos registros de aberraciones en el plumaje para varias especies de aves en Venezuela. Revista Venezolana de Ornitología, 6, 68-73.

Schlatter, R.P., y Riveros, G.M. (1987). Historia natural del archipiélago Diego Ramírez, Chile. Serie Científica INACH, 47, 87-112.

van Grouw, H. (2006). Not every white bird is an albino: sense and nonsense about colour aberrations in birds. Dutch Birding, 28, 79-89.

van Grouw, H. (2013). What Colour is that bird? The causes and recognition of common colour aberrations in birds. British Birds, 106, 17-29 
Apéndice: Afiliación de cada autor

Número

afiliación

Nombre de la institución y/o organización Afiliación

1

Unión de Ornitólogos de Chile y Programa de Conservación Biocultural Subantártica,

Parque Etnobotánico Omora.

2 Programa de Conservación Biocultural Subantártica, Parque Etnobotánico Omora

\begin{tabular}{|c|c|}
\hline Autor & Afiliación \\
\hline Juan Torres-Mura & 1 \\
\hline Omar Barroso & 2 \\
\hline
\end{tabular}

